\title{
MicroRNA-34c is associated with emphysema severity and modulates SERPINE1 expression
}

\author{
Santiyagu M Savarimuthu Francis ${ }^{1,2}$, Morgan R Davidson ${ }^{1,2}$, Maxine E Tan², Casey M Wright ${ }^{1,2}$, Belinda E Clarke ${ }^{3}$,
} Edwina E Duhig ${ }^{3}$, Rayleen V Bowman ${ }^{1,2}$, Nicholas K Hayward ${ }^{2,4}$, Kwun M Fong ${ }^{1,2}$ and lan A Yang ${ }^{1,2}$

\begin{abstract}
Background: MicroRNAs (MiRNA) are small non-coding RNAs that regulate gene expression. The aim of this study was to identify miRNAs differentially expressed between mild and moderately emphysematous lung, as well as their functional target mRNAs. Resected lung from patients with COPD undergoing lung cancer surgery was profiled using miRNA (Agilent Human miRNA profiler G4470 V1.01) and mRNA (OperonV2.0) microarrays. Cells of lung origin (BEAS-2B and HFL1) were profiled using mRNA microarrays (Illumina HumanHT-12 V3) after in vitro manipulation.

Results: COPD patients had mean (SD) age 68 (6) years, FEV 72 (17)\% predicted and gas transfer (KCO) 70 (10)\% predicted. Five miRNAs (miR-34c, miR-34b, miR-149, miR-133a and miR-133b) were significantly down-regulated in lung from patients with moderate compared to mild emphysema as defined by gas transfer $(p<0.01)$. In vitro upregulation of miR-34c in respiratory cells led to down-regulation of predicted target mRNAs, including SERPINE1, MAP4K4, ZNF3, ALDOA and HNF4A. The fold change in ex-vivo expression of all five predicted target genes inversely correlated with that of miR-34c in emphysematous lung, but this relationship was strongest for SERPINE1 $(p=0.05)$.

Conclusion: Differences in miRNA expression are associated with emphysema severity in COPD patients. MiR-34C modulates expression of its putative target gene, SERPINE1, in vitro in respiratory cell lines and ex vivo in emphysematous lung tissue.
\end{abstract}

Keywords: Chronic obstructive pulmonary disease, microRNA, miR-34c, Microarray

\section{Background}

MicroRNAs (miRNAs) are regulators that modulate transcription and translation of target mRNAs. There are over 1,000 miRNAs encoded within the human genome and these miRNAs are estimated to target $\sim 60 \%$ of genes. The discovery of miRNAs has introduced a new level of complexity to be considered for every biological process. MiRNAs are implicated in normal biological functioning, including development, differentiation, homeostasis and apoptotic cell death [1]. Their dysregulation leads to disease processes including malignancy [2-5], inflammation $[6,7]$ and heart disease [8].

MiRNAs are mediators of inflammation [9], an important mechanism driving chronic obstructive pulmonary

\footnotetext{
* Correspondence: ss.francis@uqconnect.edu.au

'Department of Thoracic Medicine, The Prince Charles Hospital, Brisbane, QLD 4032, Australia

${ }^{2}$ School of Medicine, The University of Queensland, Brisbane, QLD 4072, Australia

Full list of author information is available at the end of the article
}

disease (COPD) development and progression. Several miRNAs have been implicated in lung disease [10]. Exposure to cigarette smoke extract (CSE), diesel exhaust particles (DEP) and lipopolysaccharide (LPS) induce changes in lung expression of several miRNAs including $m i R-26 b$, miR-31, Let-7a and miR-192 [10]. MiRNAs associated with asthma include miR-21, miR-126, miR-133a, miR$148 a / b$ and $m i R-152$ [11]. Other miRNAs are associated with the inflammation observed in idiopathic pulmonary fibrosis (IPF) [12] and cystic fibrosis [13], and many studies implicate miRNAs in the pathogenesis of lung cancer. Collectively these studies demonstrate that miRNAs are expressed in the lung and are involved in the pathogenesis of lung disease.

COPD prevalence is continually increasing and it is predicted to become the third leading cause of death worldwide by $2020[14,15]$. COPD is under-diagnosed, since patients are most often diagnosed only when the disease is advanced, too late for prevention, and when there is no curative intervention. Better screening to
() Biomed Central

(c) 2014 Savarimuthu Francis et al.; licensee BioMed Central Ltd. This is an open access article distributed under the terms of the Creative Commons Attribution License (http://creativecommons.org/licenses/by/2.0), which permits unrestricted use, distribution, and reproduction in any medium, provided the original work is properly cited. 
detect early disease and targeted therapies to prevent progression to severe COPD are urgently needed to improve COPD outcomes. Genomic tools provide an opportunity to improve our understanding of the pathogenesis of COPD and translate this to enhanced clinical management [16]. Current understanding of COPD pathogenesis is that lung injury by inhaled tobacco results in depletion of lung alveolar tissue, large and small airway inflammation, fibrosis of small airways and mucus hypersecretion contributing to small airway obstruction. Inflammation is the main cause of lung tissue destruction and provokes defense mechanisms associated with tissue repair [17].

Given the role of miRNAs in lung inflammation and the lung's response to inhaled toxins, miRNAs are likely to be involved in pathogenesis and/or progression of COPD. Furthermore, miRNAs regulate mRNA expression, and mRNA (transcriptome) profiles relevant to COPD pathogenesis have been identified [18-23]. MiRNA profiles in lung from patients with COPD have shown differences in miRNA expression in relation to susceptibility to COPD $[24,25]$, when comparing moderate/severe COPD patients with non-COPD subjects [24,25]. Here we aimed to identify lung miRNAs associated with COPD severity, and demonstrate miRNA regulation of genes that may be implicated in the progression of COPD.

\section{Methods}

\section{Participant selection}

This study was approved by the Human Research Ethics Committees of The University of Queensland and The Prince Charles Hospital (TPCH). Lung tissue samples from 29 patients with COPD undergoing curative resection for lung cancer were selected from the TPCH tissue bank. Written informed consent was obtained from all patients prior to surgery. Detailed information on subject selection, and inclusion and exclusion criteria are listed elsewhere [18]. Briefly, smokers with at least a 20 pack year history who quit smoking at least 10 months prior to surgery, not currently on inhaled or oral steroids, and without other lung pathology that may confound spirometry measurements, were considered. All had $\mathrm{FEV}_{1} / \mathrm{FVC}$ (or $\mathrm{FEV}_{1} / \mathrm{VC}$ ) ratio $<0.70$, indicating the presence of COPD. Twenty-nine former smokers with COPD (the 'TPCH-KCO set') were selected and classified by gas transfer measurements (single breath carbon monoxide diffusion coefficient, $\mathrm{KCO}$ ) as "mild" emphysema ( $>75 \%$ predicted $\mathrm{KCO})$ and "moderate" emphysema (40-75\% predicted $\mathrm{KCO})$.

\section{miRNA isolation, hybridization and data extraction}

Total RNA was isolated from 30-45 mg of non-tumour lung tissue using Trizol (Invitrogen). DNA was removed using an Ambion DNA-free ${ }^{\mathrm{Tn}}$ kit (Ambion, Foster City, CA, USA) following the manufacturer's instructions. The resulting total RNA was cleaned using the DNeasy miRNA clean up kit (QIAGEN, Valencia, CA, USA) following manufacturer's instructions. Purified RNA was quality checked using a Bioanalyzer Agilent Technologies, Santa Clara, CA, USA), based on the presence of miRNAs determined using a small RNA analysis kit and distinct $18 \mathrm{~S}$ and $28 \mathrm{~S}$ peaks determined by an mRNA analysis kit. Agilent human miRNA oligo arrays $(8 \times 15 \mathrm{~K}$ array, Part No. G4470A, Agilent Technologies) based on miRBase V9.1 were used. This array contains 15,744 elements representing 470 human miRs and 64 viral miRs. The experiments were performed according to the manufacturer's instructions. Briefly, $100 \mathrm{ng}$ of total RNA from lung tissue was dephosphorylated using calf intestine alkaline phosphotase to create sticky ends for ligation of the fluorescent dye pCp-Cy3 and T4 RNA ligase (GE Healthcare, Little Chalfont, England). The resulting labeled RNA was purified using Micro-bio spin 6 columns (Bio-Rad, Hercules, CA, USA) to remove unlabeled dye and then hybridized to the Agilent microarray slide for 20 hours at $55^{\circ} \mathrm{C}$. The slides were washed to remove any unbound RNA and images were captured using Agilent microarray scanner. Raw features were filtered for spot quality, averaged and extracted using Agilent feature extraction software. The missing values were filled in using the K-nearest neighbor algorithm in Avadis (Strand Life Sciences, Bangalore, India). Study design for microarray experiments satisfied the MIAME guidelines (http://www.mged. org/Workgroups/MIAME/miame_checklist.html). The referred data has been deposited in the Gene Expression Omnibus (GEO) (GSE33338 http://www.ncbi.nlm.nih.gov/ $\mathrm{gds} /$ ?term=GSE33338).

\section{Candidate miRNA selection and technical validation using qRT-PCR}

miRNAs differentially expressed between mild and moderate classes of emphysema lungs were identified by class comparison analysis in BRB-ArrayTools V4.2 (developed by Dr Richard Simon and Amy Peng Lam, freely accessible at http://linus.nci.nih.gov/BRB-ArrayTools.html). A $P$ value of $<0.01$ and false discovery rate (FDR) of $<0.05$ were used as selection criteria for significance. Two miRNAs were randomly chosen for technical validation by quantitative reverse transcriptase polymerase chain reaction (qRT-PCR) using TaqMan microRNA assays (Applied Biosystems, Foster City, CA, USA). The geometric mean (GeNorm [26]) of two small RNA housekeepers, U6 and RNU48, were used for normalization [26,27].

\section{Identifying genes modulated by miR-34c using in vitro techniques}

mRNAs targets of altered miRNA expression in commonly used lung cell lines were identified using in vitro techniques. 


\section{Cell lines}

Commercial lung cell lines BEAS-2B [28] (CRL-9609, a human bronchial epithelial cell line) and HFL1 [29] (CCl-153, a human fetal lung fibroblast cell line) were purchased from ATCC (Virginia, USA). The cell lines were grown as per the supplier's recommendations. BEAS-2B and HFL1 were cultured in RPMI and DMEM, respectively, supplemented with antibiotics and 10\% FCS and incubated in $5 \% \mathrm{CO}_{2}$.

\section{Transfection conditions}

A microRNA $(m i R-34 c)$ that technically validated and displayed high fold change difference between emphysema classes was chosen for this purpose. Pre-miR ${ }^{\mathrm{mm}}$ miRNA precursor molecules (Invitrogen by Life Sciences, Carlsbad, CA) for $m i R-34 c-5 p$ were used to increase the expression of the $m i R-34 c$ in HFL1 and BEAS-2B lung cells and the expected increase in expression of $m i R-34 c$ was confirmed using TaqMan microRNAs assays (Invitrogen by Life Sciences, Carlsbad, CA). The $-5 p$ isoform of $m i R-34 c-5 p$ represents the $5^{\prime}$ arm of the hairpin precursor of the mature miRNA from which the mature sequence has been excised. The probe sequence represented on the microarray was derived from the $m i R-34 c-5 p$ sequence. Optimal conditions were tested and selected based on the manufacturer's instructions. Briefly 50,000 cells were transiently transfected with $20 \mathrm{nM}$ pre-miR precursor molecules and NeoFX transfection reagent for 24 hours. The transfection was conducted twice in triplicate each time on two different days two weeks apart. The triplicates were combined for the arrays to provide enough total RNA for the assay.

\section{mRNA isolation, hybridization and data extraction}

Total RNA was extracted and purified from cell lines, BEAS-2B and HFL1, after transfection, using RNeasy Mini kit and RNAse free DNAse kit (QIAGEN, Hilden, Germany). Microarray expression profiling was conducted on the purified RNA using Illumina HT12V3 whole genome gene expression arrays, according to the manufacturer's instructions. The array contains 48,000 elements representing over 25,000 annotated genes from the RefSeq (Build 36.2) and Unigene databases (Build 199). Element features were extracted using the gene expression module of the BeadStudio V1.1.1 software (GenomeStudio, Illumina, Hayward, CA). Raw features were normalized to the 75th percentile of all elements in GeneSpring GX V9 (Agilent Technologies, CA, USA). Missing values were filled in using the K-nearest neighbor algorithm in Avadis (Strand LifeSciences, Bangalore, India). Differentially expressed genes were identified using class comparison analysis in BRB-ArrayToolV4.2.

\section{Identification of $\mathrm{miR}-34 \mathrm{c}$ predicted targets}

The genes differentially expressed between $m i R-34 c$ transfected and non-transfected cells were compared to the predicted targets of miR-34c from the TargetScan and PicTar databases. Candidate target genes whose expression were negatively correlated to that of $m i R-34 \mathrm{c}$ in vitro (in cell lines) and ex vivo (in lung of TPCH-KCO and Spira et al. [19] subjects) were identified. Therefore, identified genes were biologically validated in the original TPCH-KCO [18] samples (used to develop microRNA profiles) by qRT-PCR using pre-designed gene expression assays (Applied Biosystems, Foster City, CA, USA). The raw expression was normalised to the house-keeping gene, GAPDH.

\section{Results}

Identification of miRNAs associated with emphysema severity using microarrays and confirmation by qRT-PCR Agilent human miRNA oligo $8 \times 15 \mathrm{~K}$ array experiments were conducted on samples of lung from patients with mild ( $>75 \%$ predicted $\mathrm{KCO}, \mathrm{n}=9$ ) or moderate emphysema (40 to $75 \%$ predicted $K C O, n=20$ ). Demographic data are shown in Table 1. After preprocessing of the raw signals, 228 miRNAs were detected in at least $50 \%$ of the lung samples. Duplicate array data for these 228 miRNAs were highly correlated $\left(r^{2}=0.98\right.$, Additional file 1: Figure S1). Class comparison identified five miRNAs ( $m i R-34 c, m i R$ $34 b, m i R-149, m i R-133 a$ and $m i R-133 b)$ that were significantly differentially expressed between mild and moderate emphysema ( $<<0.01$, Additional file 1: Figure S2 \& Table 2). All five were expressed at lower levels in lung from patients with moderate emphysema, compared with lung from patients with mild emphysema. In this cohort, expression of three of these miRNAs (miR-149, miR-133a and miR-133b) correlated with functional measurement of emphysema severity (KCO, Figure 1). MiR-34c expression exhibited the greatest difference between groups with 0.3 fold lower expression in the moderate severity group. qRT-PCR confirmed similar fold differences in expression to microarray results for the two miRNAs, $m i R-34 c$ and $m i R-133 a$ tested (Additional file 1: Figure S3). A flow diagram describing the methods is shown in Additional file 1: Figure S4.

\section{Effect of miR-34c-5p over-expression on its predicted mRNA targets in respiratory cells in vitro using gene expression microarrays}

To identify mRNA targets regulated by $m i R-34 c$ in lung cells, we transfected $m i R-34 c-5 p$ precursor into BEAS-2B and HFL1 cells, and demonstrated the expected $95-99 \%$ increased expression of $m i R-34 c$ by qRT-PCR (data not shown). Illumina HumanHT12 V3 transcriptome microarrays were then used to examine the expression of mRNAs in miR-34c-transfected cells, relative to cells transfected with scrambled sequence controls. Class comparison analysis in BRB-ArrayTools V4.2 identified 2,152 elements 
Table 1 Demographics of the TPCH-KCO set ( $\mathrm{n}=29$ patients) used in microRNA microarrays

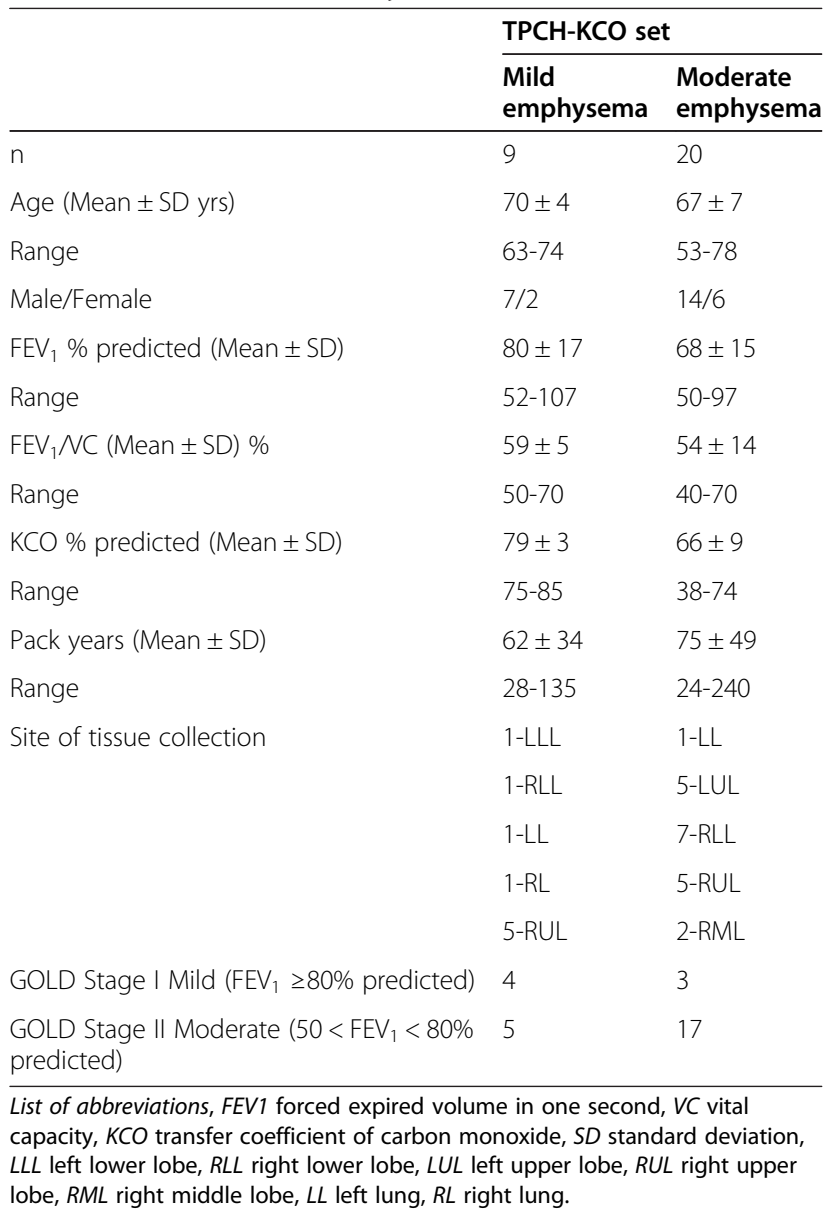

(48 expected by chance) affected by over-expression of miR-34c $(p<0.001)$ in BEAS-2B and HFL1 cell lines, the vast majority of which $(90 \%)$ were down-regulated by elevated miR-34c levels. Among the 2,152 genes, fifty were predicted as miR-34c targets by the databases Pictar and TargetScan-and they were significantly down-regulated ( $>1.8$ fold down-regulation) in the transfectants. We next examined ex vivo expression patterns of the genes found to be $m i R-34 c$ responsive in vitro. Of the fifty genes, thirtythree were represented in the filtered TPCH-KCO dataset [18] and thirty-two in the dataset of Spira et al. [19].
In both of these datasets, five genes (MAP4K4, SERPINE1, ALDOA, HNF4A and ZNF3) were expressed at higher levels in lung from patients with moderate or severe emphysema compared with lung from normal subjects or subjects with mild emphysema, whereas expression of $m i R-34 c$ was reciprocal (Figure 2), consistent with the possibility that these five genes may be regulated by miR-34c in emphysema. In the 29 ex vivo lung samples used for miRNA microarray profiling, qRTPCR confirmed that expression of both SERPINE1 (fold-change $>2.2$ ) and HNF4A (fold-change >1.3) had generally higher expression in association with lower miR-34c expression; however only SERPINE1 $(p=0.05)$ was significantly differentially expressed between mild and moderate emphysema (Figure 3) in this cohort, and it was correspondingly upregulated in more severe disease in both TPCH-KCO (fold change $>1.2, p<0.05$ ) and Spira (fold change $>1.13$ ) datasets (Figure 2).

\section{Discussion}

MiRNAs regulate the expression of genes involved in biological processes relevant to the progression of chronic lung disease, including cellular stress, cell differentiation and apoptosis [30-32]. Here, we aimed to identify miRNAs (and their target mRNAs) associated with emphysema severity in COPD patients. Five miRNAs were identified to be significantly down-regulated in lung from patients with moderate emphysema, compared with lung of mild emphysema patients. In addition, a negative correlation was noted in the expression of these miRNAs and their putative target mRNAs ex vivo. Furthermore, increasing the expression of $m i R-34 c$ (the miRNA with the largest fold change between the mild and moderate emphysema patient groups) in respiratory cells resulted in decreased expression of predicted mRNA targets in vitro, providing additional functional expression data. Our data suggest modulation of SERPINE1 by miR-34c in vitro and ex vivo. Dysregulation of SERPINE1 by miR-34c could therefore be a potential mechanism involved in emphysema severity and progression. To our knowledge, this is the first study to compare miRNA profiles in the lungs of COPD patients based on emphysema severity.

Table 2 Demographics of miRNAs significantly downregulated in the moderate emphysema patients compared with mild emphysema patients

\begin{tabular}{llllll}
\hline Unique id & p-value & $\begin{array}{l}\text { False discovery } \\
\text { rate (FDR) }\end{array}$ & $\begin{array}{l}\text { Geometric mean of } \\
\text { class 1 (moderate) }\end{array}$ & $\begin{array}{l}\text { Geometric mean of } \\
\text { class 2 (mild) }\end{array}$ & $\begin{array}{l}\text { Fold-change } \\
\text { hsa-miR-149 }\end{array}$ \\
hsa-miR-133a & 0.001 & 0.019 & 13.60 & 24.24 & 0.56 \\
hsa-miR-133b & 0.002 & 0.019 & 35.66 & 64.71 & 0.55 \\
hsa-miR-34c & 0.003 & 0.019 & 49.72 & 90.43 & 0.55 \\
hsa-miR-34b & 0.006 & 0.034 & 57.53 & 158.14 & 0.36 \\
\hline
\end{tabular}



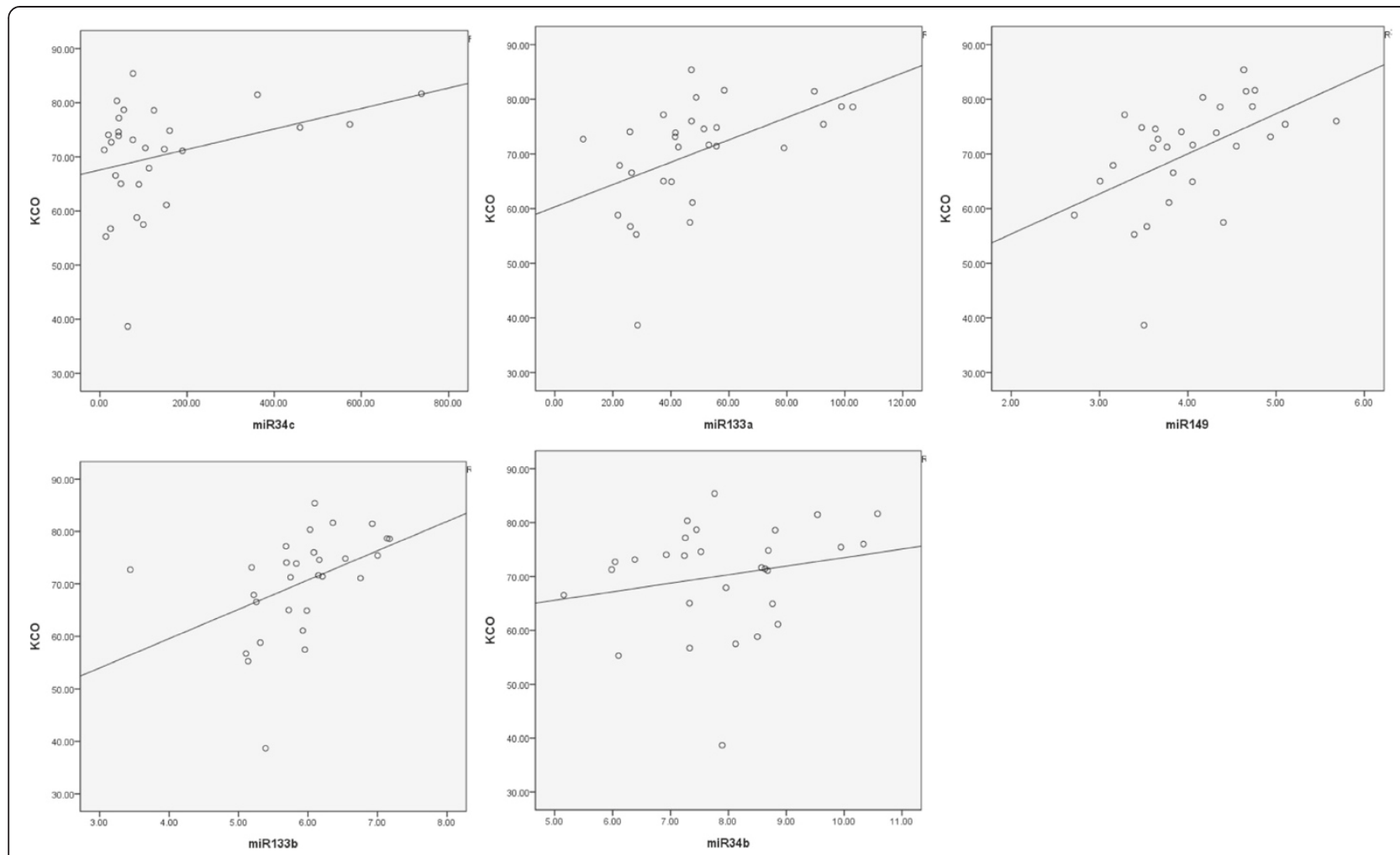

\begin{tabular}{|l|l|l|l|}
\hline miRNA & $\boldsymbol{p}$ & $\mathrm{R}^{2}$ & $\begin{array}{l}\text { Fold change (ratio } \\
\text { moderate/mild } \\
\text { emphysema) }\end{array}$ \\
\hline miR-34c & $\mathbf{0 . 0 7}$ & $\mathbf{0 . 1 1}$ & $\mathbf{0 . 3 6}$ \\
\hline miR-133a & $0.007^{* *}$ & 0.239 & 0.55 \\
\hline miR-149 & $0.005^{* *}$ & 0.255 & 0.56 \\
\hline miR-133b & $0.02^{*}$ & 0.185 & 0.55 \\
\hline miR-34b & 0.276 & 0.044 & 0.42 \\
\hline
\end{tabular}

Figure 1 Correlation plots of the five candidate miRNAs and emphysema status. The expression of the differentially expressed microRNAs for the 29 patients and their emphysema status (KCO\% predicted corrected for hemoglobin) is shown. The correlation of miR34c expression determined by microarrays in lung tissues to its consequent KCO measurements were not significant $(p>0.05)$. However this miRNA was chosen for cell line studies as it portrayed siginificant difference in gene expression between patient groups i.e. moderate vs mild emphysema lungs. MiRNA levels ofthe 29 samples are drawn on the $\mathrm{X}$-axis, $\mathrm{KCO} \%$ predicted on the left $\mathrm{Y}$-axis and microRNA expression on the right $\mathrm{Y}$-axis. * indicates a correlation of significance $p<0.05$ and ${ }^{* *}$ indicates a correlation of significance $p<0.01$.

A major strength of this study was the ability to correlate miRNA-mediated mRNA regulation in the same lung samples. It is known that miRNAs mediate gene silencing through translational inhibition and mRNA degradation. In addition, target mRNA is degraded in more than one third of genes that display translational repression [33]; hence we have focused on studying changes in mRNA expression as a consequence of altered miRNA expression. MiRNAs that are down-regulated in a certain disease state (e.g. moderate emphysema, compared to mild emphysema) would be expected to upregulate expression of their predicted mRNAs, in an orchestrated process of epigenetic regulation. This relationship of miRNA and mRNA expression has been demonstrated previously in airways from COPD patients [25] but not in peripheral lung tissue, which we used in this current 


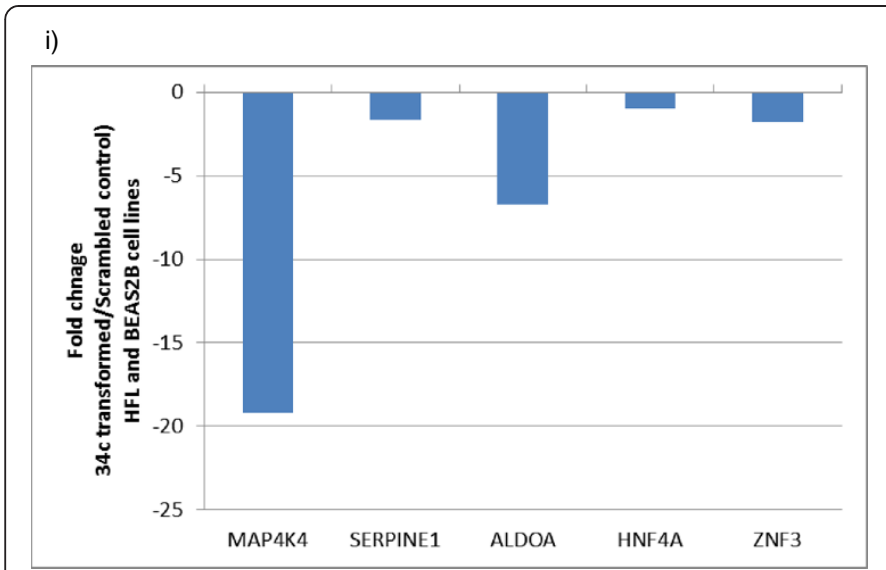

mRNA expression of the fives genes in in-vitro in respiratory cell-lines ii)

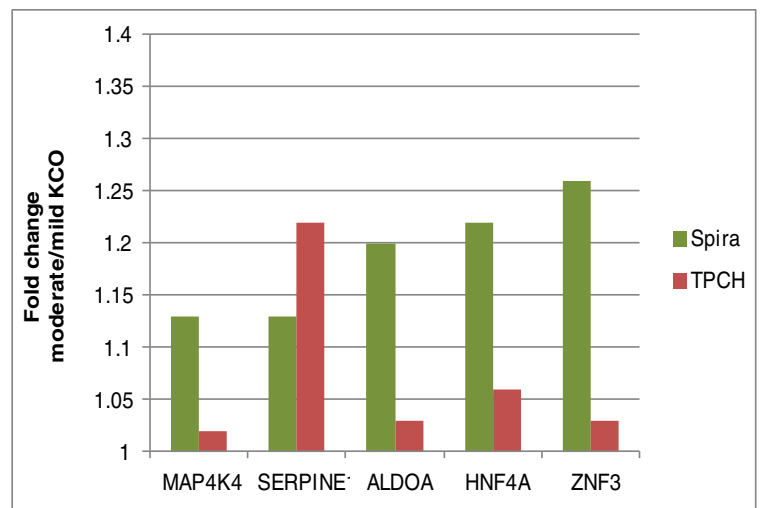

mRNA expression of the fives genes in ex-vivo lung tissue samples where the initial miRNAs were recorded

Figure 2 Histogram of in vitro (i) and ex vivo (ii) expression of genes predicted and modulated by miR-34c over-expression in BEAS-2B and HFL1 cell lines. Ratio of moderate to mild gene expression of emphysema patients are shown on the Y-axis and the genes on the X-axis.

study. Of the miRNAs identified as differentially expressed in lung between moderate vs mild emphysema patients in this study, miR-34c is particularly relevant to human lung disease, as its expression is increased during normal lung development [34]. We found decreased expression of $m i R-34 c$ in lungs of patients with moderate emphysema, compared to mild emphysema. Van Pottelberge et al. also found greater than three-fold downregulation of $m i R-34 c$ in sputum from smokers with COPD, compared to those without COPD, and a direct correlation between $m i R-34 c$ expression and percent predicted $\mathrm{FEV}_{1}$ [24]. Their

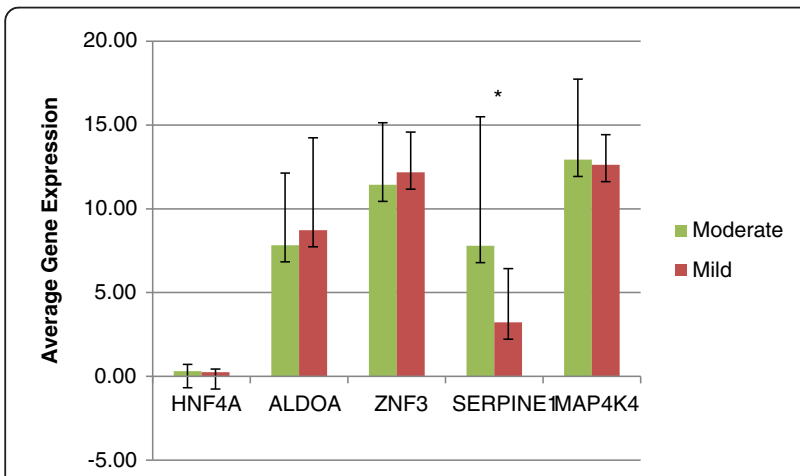

Figure 3 Histogram of qRT-PCR measurements of miR-34c regulated targets in TPCH-KCO dataset. Genes differentially expressed between transformed and scrambled sequence controls in respiratory cell lines were independently tested in TPCH-KCO dataset using qRT-PCR. This step minimizes false positives and ensures that authentic genes are discovered. Average gene expression in mild and moderate emphysema lungs are represented in the $Y$-axis and genes on the X-axis. SERPINE1 was significantly upregulated in emphysema lungs. HNF4 was also upregulated but did not attain significance. findings support our study, since there was downregulation of $m i R-34 c$ expression in their COPD patients, compared to controls, and also down-regulation in our moderate emphysema patients, compared to mild emphysema, indicating a similar continuum of expression with increasing disease severity. In the setting of reduced $m i R$ $34 c$ expression in moderate emphysema lung (vs mild), we were able to confirm increased gene expression of two of its predicted mRNA targets, HNF4 and SERPINE1. HNF4A (hepatocyte nuclear factor 4A) is a transcription factor that regulates the expression of cytokines involved in inflammation, as demonstrated in other organs (e.g. kidney [35]); its role in the lung is yet to be determined. SERPINE1 (Serpin Peptidase Inhibitor, Clade E (Nexin, Plasminogen Activator Inhibitor Type 1), Member 1), also known as plasminogen activator inhibitor 1 (PAI-1), is a protease inhibitor, and inhibitor of fibrinolysis. PAI-1 knockout mice demonstrate emphysema-like changes in the lung [36], so our findings of increased SERPINE1 expression in moderate emphysema (vs mild emphysema) may be unexpected, given the role of protease-antiprotease imbalance in the pathogenesis of emphysema. However, SERPINE1 is only one of a number of antiproteases, and has other roles in the lung that could be relevant to the progression of emphysema. For example, SERPINE1 has been shown to be upregulated in the lung or lung cells during hypoxia [37], exposure to lipopolysaccharide or cigarette smoke extract [38], or oxidative stress via NF- $\mathrm{kB}$ [39]. Importantly, elevated levels of PAI-1 in sputum have been observed in two separate studies of patients with COPD (compared to controls) [39,40]. Furthermore, sputum PAI-1 levels were higher in more severe COPD (compared to milder COPD), which would be in keeping with 
our findings of higher SERPINE1 expression in more severe emphysema [40]. Other candidate emphysema severity miRNAs identified in this study - miR-34b, miR-133a/ $b$ and miR-149 - have been previously implicated in the pathogenesis of lung diseases. For example, miR-34b expression was decreased in induced sputum from COPD smokers compared to non-COPD smokers [24]. Downregulation of $m i R-133 a$ is associated with increased bronchial smooth muscle contraction in patients with asthma [41]. miR-133b negatively regulates the expression of transcription factor, Pitx3 [42], which activates the dopamine receptor (DRD1), a mediator of nicotine addiction in smokers [43]. Overall, emerging evidence of the biological roles of these miRNAs supports the plausibility of their involvement in lung diseases.

A number of potential limitations of this study should be addressed. (i) A physiological measure of emphysema (gas transfer) was used to stratify patients, as in our previous study [18], rather than pathological examination of lung tissue. The reason for this was that these lung tissue samples were not inflated at harvesting, making morphological assessment of emphysema severity difficult. However, gas transfer is routinely used clinically as a measure of emphysema severity in COPD patients. (ii) Although a relatively small number of samples was used (from 29 patients), the FDRs of the 5 miRNAs were all $<0.05$; furthermore, the two miRNAs tested (miR-34c and $m i R-133 a$ ) were technically validated independently using qRT-PCR. (iii) No overlap was found between the $m i R-34 c$ targets that were enriched in ex vivo lung samples and in vitro cell lines. It should be noted that the ex vivo study used Operon microarrays which represented only 14,000 genes. Furthermore, our in vitro study used commercial cell lines, and all of our COPD lung samples were from patients who also had lung cancer. Despite these potential limitations, we found that the expression of five candidate genes in vitro correlated with ex vivo profiles in both in-house [18] and external datasets (Spira et al. [19]).

\section{Conclusion}

In summary, miRNA expression is associated with increasing emphysema severity in COPD patients. Increasing $m i R-34 c$ expression in vitro in respiratory cell lines decreased the expression of its predicted targets, consistent with miRNA-mediated regulation of mRNA. Additionally, we have shown by qRT-PCR that SERPINE1 expression is increased in lung from patients with increasing severity of emphysema, in whom miR-34c expression is low. Future studies should aim to directly demonstrate $m i R-34 c$ mediated SERPINE1 regulation in emphysema and evaluate the pathogenetic role of other miRNAs, with the goal of identifying potentially important targets for more effective treatment of COPD.

\section{Availability of supporting data}

The data sets supporting the results of this article are available in the Gene Expression Omnibus (GEO), identified as GSE33338 (http://www.ncbi.nlm.nih.gov/gds/? term $=$ GSE33338).

\section{Additional file}

\begin{abstract}
Additional file 1: Figure S1. Graphical comparison of technical replicates used in the miRNA microarrays on Agilent Human miRNA profiler. Two lung samples were randomly chosen to be repeated on different array on different days to evaluate the reproducibility of the arrays. Raw signal intensity of all 550 miRNAs is shown for the replicate samples. Figure S2. Dendrogram of the i) 228 filtered miRNAs used in the class comparison analysis and ii) the five miRNAs significantly differentially expressed between mild and moderate emphysema $(p<0.01)$. The red and green colours indicate miRNAs over and under expressed in the mild compared with moderate emphysema patients, respectively. The yellow and blue bars indicate mild and moderate emphysema samples, respectively. Figure S3. Technical validation of microRNA expression for two microRNAs. A) Histogram of microRNA expression measured by qRT-PCR and microarray. The expression is shown as the ratio of moderate to mild emphysema patients on the Y-axis for both methods. B) Correlation plots of microRNA expression measured by qRT-PCR versus microarrays. Figure S4. Analytical flow diagram describing the miRNA microarray data analysis, miRNA identification, miRNA-mRNA target correlation and in vitro validation.
\end{abstract}

\section{Competing interests}

The authors declare that they have no competing interests.

\section{Authors' contributions}

SS carried out all the experiments, analysis and drafted the manuscript. MD participated in the initial planning of experiments and analysis of results. MT helped with cell line maintenance and gene expression arrays. CW helped with gene expression arrays. BC and ED collected and graded all lung tissue specimens used in the study. RB, NK, KF and IY participated in the planning of the study design, experiments and analysis. IY helped with the drafting of the manuscript. All authors read and approved the final manuscript.

\section{Acknowledgments}

We acknowledge the support of the patients and staff of The Prince Charles Hospital for their participation, and The Prince Charles Hospital surgeons, pathologists, scientists and Thoracic Research Laboratory staff for their assistance with specimen collection. Funding bodies, NHMRC Biomedical Scholarship (SF), Cancer Council Queensland PhD Scholarship (MD), NHMRC Career Development Fellowship (IY), NHMRC Practitioner Fellowship (KF), NHMRC Senior Principal Research Fellowship (NH), The Prince Charles Hospital Foundation, Asthma Foundation of Queensland, Queensland Health Smart State Research Grant, and Australian Lung Foundation/Boehringer Ingelheim COPD Research Fellowship (IY).

\section{Author details}

'Department of Thoracic Medicine, The Prince Charles Hospital, Brisbane, QLD 4032, Australia. ${ }^{2}$ School of Medicine, The University of Queensland, Brisbane, QLD 4072, Australia. ${ }^{3}$ Department of Anatomical Pathology, The Prince Charles Hospital, Brisbane, QLD 4032, Australia. ${ }^{4}$ Queensland Institute of Medical Research, Brisbane, QLD 4006, Australia.

Received: 6 November 2012 Accepted: 23 January 2014

Published: 30 January 2014

\section{References}

1. Chen XM, Splinter PL, O'Hara SP, LaRusso NF: A cellular micro-RNA, let-7i, regulates Toll-like receptor 4 expression and contributes to cholangiocyte immune responses against Cryptosporidium parvum infection. $J$ Biol Chem 2007, 282(39):28929-28938. 
2. Yanaihara N, Caplen N, Bowman E, et al: Unique microRNA molecular profiles in lung cancer diagnosis and prognosis. Cancer Cell 2006, 9(3):189-198.

3. Lin PY, Yu SL, Yang PC: MicroRNA in lung cancer. Br J Cancer 2010, 103(8):1144-1148.

4. Blenkiron C, Goldstein LD, Thorne NP, et al: MicroRNA expression profiling of human breast cancer identifies new markers of tumor subtype. Genome Biol 2007, 8(10):R214.

5. Heneghan HM, Miller N, Kerin MJ: MiRNAs as biomarkers and therapeutic targets in cancer. Curr Opin Pharmacol 2010, 10(5):543-550.

6. O'Connell RM, Taganov KD, Boldin MP, Cheng G, Baltimore D: MicroRNA-155 is induced during the macrophage inflammatory response. Proc Natl Acad Sci USA 2007, 104(5):1604-1609.

7. Sonkoly E, Pivarcsi A: microRNAs in inflammation. Int Rev Immunol 2009 28(6):535-561.

8. Mann DL: MicroRNAs and the failing heart. N Engl J Med 2007, 356(25):2644-2645.

9. Sheedy FJ, O'Neill LA: Adding fuel to fire: microRNAs as a new class of mediators of inflammation. Ann Rheum Dis 2008, 67(Suppl 3):iii50-iii55.

10. Oglesby IK, McElvaney NG, Greene CM: MicroRNAs in inflammatory lung disease-master regulators or target practice? Respir Res 2010, 11:148.

11. Jiang $X$ : The emerging role of microRNAs in asthma. Mol Cell Biochem 2011, 353(1-2):35-40

12. Pandit KV, Corcoran D, Yousef $H$, et al: Inhibition and role of let-7d in idiopathic pulmonary fibrosis. Am J Respir Crit Care Med 2010, 182(2):220-9.

13. Oglesby IK, Bray IM, Chotirmall SH, et al: miR-126 is downregulated in cystic fibrosis airway epithelial cells and regulates TOM1 expression. J Immunol 2010, 184(4):1702-9.

14. Murray CJ, Lopez AD: Alternative projections of mortality and disability by cause 1990-2020: Global Burden of Disease Study. Lancet 1997, 349(9064):1498-504.

15. Lopez AD, Murray CC: The global burden of disease, 1990-2020. Nat Med 1998, 4(11):1241-3.

16. Yang IA, Francis SM: Deconstructing COPD using genomic tools. Respirology 2009, 14(3):313-7.

17. Scanlon PD: The pathogenesis and pathology of COPD: identifying risk factors and improving morbidity and mortality. Adv Stud Med 2004, 4(10A):S744-S749.

18. Savarimuthu Francis SM, Larsen JE, Pavey SJ, et al: Expression profiling identifies genes involved in emphysema severity. Respir Res 2009, 10(1):81.

19. Spira A, Beane J, Pinto-Plata $V$, et al: Gene expression profiling of human lung tissue from smokers with severe emphysema. AJRCMB 2004, 31(6):601-10.

20. Ning W, Li CJ, Kaminski N, et al: Comprehensive gene expression profiles reveal pathways related to the pathogenesis of chronic obstructive pulmonary disease. Proc Natl Acad Sci U S A 2004, 101(41):14895-900.

21. Wang IM, Stepaniants S, Boie $Y$, et al: Gene expression profiling in patients with chronic obstructive pulmonary disease and lung cancer. Am J Respir Crit Care Med 2008, 177(4):402-11.

22. Bhattacharya S, Srisuma S, Demeo DL, et al: Molecular biomarkers for quantitative and discrete COPD phenotypes. AJRCMB 2009, 40(3):359-67.

23. Savarimuthu Francis SM, Larsen JE, Pavey SJ, et al: Genes and gene ontologies common to airflow obstruction and emphysema in the lungs of patients with COPD. PLOS ONE 2011, 6(3):e17442.

24. Van Pottelberge GR, Mestdagh P, Bracke KR, et al: MicroRNA expression in induced sputum of smokers and patients with chronic obstructive pulmonary disease. Am J Respir Crit Care Med 2011, 183(7):898-906.

25. Ezzie ME, Crawford M, Cho JH, et al: Gene expression networks in COPD: microRNA and mRNA regulation. Thorax 2012, 67(2):122-31.

26. Vandesompele J, De Preter K, Pattyn F, et al: Accurate normalization of real-time quantitative RT-PCR data by geometric averaging of multiple internal control genes. Genome Biol 2002, 3(7):RESEARCH0034.

27. Zeskind JE, Lenburg ME, Spira A: Translating the COPD Transcriptome: Insights into Pathogenesis and Tools for Clinical Management. Proc Am Thorac Soc 2008, 5(8):834-41.

28. Chen ZH, Kim HP, Sciurba FC, et al: Egr-1 regulates autophagy in cigarette smoke-induced chronic obstructive pulmonary disease. PLOS ONE 2008, 3(10):e3316.
29. Carnevali S, Petruzzelli S, Longoni B, et al: Cigarette smoke extract induces oxidative stress and apoptosis in human lung fibroblasts. Am J Physiol Lung Cell Mol Physiol 2003, 284(6):L955-63.

30. Cheng AM, Byrom MW, Shelton J, Ford LP: Antisense inhibition of human miRNAs and indications for an involvement of miRNA in cell growth and apoptosis. Nucleic Acids Res 2005, 33(4):1290-7.

31. Cimmino A, Calin GA, Fabbri M, et al: miR-15 and miR-16 induce apoptosis by targeting BCL2. Proc Natl Acad Sci U S A 2005, 102(39):13944-9.

32. Babar IA, Slack FJ, Weidhaas JB: miRNA modulation of the cellular stress response. Future Oncol 2008, 4(2):289-98.

33. Baek D, Villen J, Shin C, Camargo FD, Gygi SP, Bartel DP: The impact of microRNAs on protein output. Nature 2008, 455(7209):64-71

34. Bouhallier F, Allioli N, Lavial F, et al: Role of miR-34c microRNA in the late steps of spermatogenesis. Rna 2010, 16(4):720-31.

35. Wang Z, Bishop EP, Burke PA: Expression profile analysis of the inflammatory response regulated by hepatocyte nuclear factor 4 alpha. BMC Genomics 2011, 12:128.

36. Hu H, Zhao $Y$, Xiao $Y$, Zhang $R$, Song $H$ : Disruption of plasminogen activator inhibitor-1 gene enhances spontaneous enlargement of mouse airspace with increasing age. Tohoku J Exp Med 2010, 222(4):291-6.

37. Muth M, Theophile K, Hussein $K_{1}$ Jacobi C, Kreipe H, Bock O: Hypoxiainduced down-regulation of microRNA-449a/b impairs control over targeted SERPINE1 (PAI-1) mRNA - a mechanism involved in SERPINE1 (PAI-1) overexpression. J Trans/ Med 2010, 8:33.

38. Xu X, Wang H, Wang Z, Xiao W: Plasminogen activator inhibitor-1 promotes inflammatory process induced by cigarette smoke extraction or lipopolysaccharides in alveolar epithelial cells. Exp Lung Res 2009, 35(9):795-805

39. To M, Takagi D, Akashi $K$, et al: Sputum plasminogen activator inhibitor-1 elevation by oxidative stress-dependent nuclear factor-kappaB activation in COPD. Chest 2013, 144(2):515-21.

40. Jiang $Y$, Xiao $W$, Zhang $Y$, Xing $Y$ : Urokinase-type plasminogen activator system and human cationic antimicrobial protein 18 in serum and induced sputum of patients with chronic obstructive pulmonary disease. Respirology 2010, 15(6):939-46.

41. Chiba Y, Misawa M: MicroRNAs and their therapeutic potential for human diseases: MiR-133a and bronchial smooth muscle hyperresponsiveness in asthma. J Pharmacol Sci 2010, 114(3):264-8.

42. Sanchez-Simon FM, Zhang XX, Loh HH, Law PY, Rodriguez RE: Morphine regulates dopaminergic neuron differentiation via miR-133b. Mol Pharmacol 2010, 78(5):935-42.

43. Novak G, LeBlanc M, Zai C, et al: Association of polymorphisms in the BDNF, DRD1 and DRD3 genes with tobacco smoking in schizophrenia. Ann Hum Genet 2010, 74(4):291-8.

doi:10.1186/1471-2164-15-88

Cite this article as: Savarimuthu Francis et al: MicroRNA-34c is associated with emphysema severity and modulates SERPINE1 expression. BMC Genomics 2014 15:88

\section{Submit your next manuscript to BioMed Central and take full advantage of:}

- Convenient online submission

- Thorough peer review

- No space constraints or color figure charges

- Immediate publication on acceptance

- Inclusion in PubMed, CAS, Scopus and Google Scholar

- Research which is freely available for redistribution 